\title{
Flows of Mellin transforms with periodic integrator
}

Article

Accepted Version

Hilberdink, T. (2011) Flows of Mellin transforms with periodic integrator. Journal de Theorie des Nombres de Bordeaux, 23. pp. 455-469. ISSN 1246-7405 Available at https://centaur.reading.ac.uk/23362/

It is advisable to refer to the publisher's version if you intend to cite from the work. See Guidance on citing.

Published version at: http://jtnb.cedram.org/?lang=en

Publisher: Société Arithmétique de Bordeaux

All outputs in CentAUR are protected by Intellectual Property Rights law, including copyright law. Copyright and IPR is retained by the creators or other copyright holders. Terms and conditions for use of this material are defined in the End User Agreement.

\section{www.reading.ac.uk/centaur}

\section{CentAUR}

Central Archive at the University of Reading

Reading's research outputs online 


\title{
Flows of Mellin transforms with periodic integrator
}

\author{
Titus Hilberdink \\ Department of Mathematics, University of Reading, Whiteknights, \\ PO Box 220, Reading RG6 6AX, UK; t.w.hilberdink@reading.ac.uk
}

\begin{abstract}
We study Mellin transforms $\hat{N}(s)=\int_{1-}^{\infty} x^{-s} d N(x)$ for which $N(x)-x$ is periodic with period 1 in order to investigate 'flows' of such functions to Riemann's $\zeta(s)$ and the possibility of proving the Riemann Hypothesis with such an approach. We show that, excepting the trivial case where $N(x)=x$, the supremum of the real parts of the zeros of any such function is at least $\frac{1}{2}$.

We investigate a particular flow of such functions $\left\{\hat{N}_{\lambda}\right\}_{\lambda \geq 1}$ which converges locally uniformly to $\zeta(s)$ as $\lambda \rightarrow 1$, and show that they exhibit features similar to $\zeta(s)$. For example, $\hat{N}_{\lambda}(s)$ has roughly $\frac{T}{2 \pi} \log \frac{T}{2 \pi}-\frac{T}{2 \pi}$ zeros in the critical strip up to height $T$ and an infinite number of negative zeros, roughly at the points $\lambda-1-2 n(n \in \mathbb{N})$.
\end{abstract}

2010 AMS Mathematics Subject Classification: 11M41, 30C15.

Keywords and phrases: Zeros of Mellin transforms, Lindelöf function.

\section{Introduction}

One idea of approaching the Riemann Hypothesis $(\mathrm{RH})$ is to construct a sequence or a flow of holomorphic functions converging to $\zeta(s)$, uniformly on compact subsets of $\mathbb{C} \backslash\{1\}$ in such a way that all the functions in the sequence have no zeros $\operatorname{in}^{1} H_{\frac{1}{2}}$. Then by Hurwitz's Theorem on the zeros of the limit function, $\mathrm{RH}$ would follow. Less stringently, we would only require that there are no zeros in half-planes converging to $H_{\frac{1}{2}}$. To make it worthwhile, it should be easier to locate the zeros of the sequence than of $\zeta(s)$ itself.

The problem with such an approach is of course how to choose your sequence or flow (if indeed this is possible). We shall restrict ourselves to Mellin transforms; i.e.

$$
\hat{N}_{\lambda}(s)=\int_{0}^{\infty} x^{-s} d N_{\lambda}(x)
$$

where $\lambda$ ranges over some interval, say $\lambda \in[0,1]$ with $N_{\lambda}(x) \rightarrow[x]$ as $\lambda \rightarrow 1$. Thus $\hat{N}_{\lambda}(s) \rightarrow \zeta(s)$.

For instance, one can imagine starting from very 'smooth' generalised primes and integers and 'flowing' to the actual primes and integers as time progresses. For example, we could start from $N_{0}(x)=x(x \geq 1)$ and zero otherwise and 'flow' to the function $N_{1}(x)=[x]$. Then $\hat{N}_{0}(s)=\frac{s}{s-1}$ 'flows' to $\hat{N}_{1}(s)=\zeta(s)$.

There are many 'natural' properties that a typical integrator $N(x)$ (or its Mellin transform) in such a flow could be assumed to have, by analogy with $[x]$ and its Mellin transform $\zeta(s)$. One property we shall assume at the outset is that $N(x)=0$ for $x<1$ and $N(1)=1$. Thus $N$ has a jump at 1 and so $\hat{N}(s)=1+\int_{1}^{\infty} x^{-s} d N(x)$, ensuring that $\hat{N}(s)$ is bounded away from zero in half-planes far enough to the right. In this paper we shall further assume that for $x \geq 1, N(x)-x$ is periodic with period 1 . (This is true for the cases $N(x)=x$ and $N(x)=[x]$

\footnotetext{
${ }^{1}$ For $\theta \in \mathbb{R}$, we denote by $H_{\theta}$ the half plane $\{s \in \mathbb{C}: \Re s>\theta\}$.
} 
mentioned above). A further property that could be considered is that $N(x)$ forms part of a generalised prime system; i.e. $N(x)=\exp _{*} \Pi(x)$ for some increasing function $\Pi(x)$, or in terms of Mellin transforms; $\log \hat{N}(s)=\hat{\Pi}(s)$. However, we shall not assume this here.

On the above assumptions $\hat{N}(s)$ has an analytic continuation to $H_{0} \backslash\{1\}$ with a simple pole at $s=1$. In fact, using the Fourier development of $N(x)-x$, we shall show (Theorem 1) that there is an analytic continuation to the rest of the complex plane as well, and furthermore $\hat{N}(s)$ satisfies a 'functional relationship' akin to the functional equation for $\zeta(s)$. As a corollary (Corollary 2) it follows that the associated Lindelöf function (see below for the definition) is at least $\frac{1}{2}-\sigma$ for $\sigma<\frac{1}{2}$, except in the case when $N(x)=x$. Denoting by $\Theta$ the supremum of the real parts of the zeros of $\hat{N}$, this further implies that $\Theta \geq \frac{1}{2}$.

In particular, this shows it is impossible to have a flow of such Mellin transforms from $\frac{s}{s-1}$ to $\zeta(s)$ in which the zeros gradually move to the right (unless RH is false).

In the final section, we discuss the zeros of a particular flow of such Mellin transforms $\left\{\hat{N}_{\lambda}\right\}_{\lambda \geq 1}$ whose integrator $N_{\lambda}$ has Fourier coefficients proportional to $n^{-\lambda}$.

\section{Some preliminaries and notation}

Let $S$ denote the space of functions $f: \mathbb{R} \rightarrow \mathbb{C}$ which are zero on $(-\infty, 1)$, right-continuous, and of local bounded variation. (See e.g. [2], pp.50-70.) For $\alpha \in \mathbb{R}$, let $S_{\alpha}=\{f \in S: f(1)=\alpha\}$.

Let $f \in S$. If $f(x)=O\left(x^{A}\right)$ for some $A$, then we define the Mellin transform by

$$
\hat{f}(s)=\int_{1-}^{\infty} x^{-s} d f(x) .
$$

This is well-defined for $\sigma=\Re s>\alpha$, where $\alpha$ is the infimum of $A$ for which $f(x)=O\left(x^{A}\right)$. Indeed, in this half-plane, $\hat{f}$ is holomorphic. Integrating by parts gives

$$
\hat{f}(s)=s \int_{1}^{\infty} \frac{f(x)}{x^{s+1}} d x .
$$

A function $F$ holomorphic in a vertical strip (except possibly at a finite number of isolated singularities) is said to be of finite order if

$$
F(\sigma+i t)=O\left(|t|^{A}\right) \quad\left(|t| \geq t_{0}, \text { some } t_{0}\right),
$$

for each $\sigma$ in the interval of the strip. As such, we may define the Lindelöf function $\mu(\sigma)$ to be the infimum of those $A$ for which the above holds. It is well-known that $\mu$ is a convex function. In our case (with $F=\hat{N}$ and $N \in S_{1}$ ), $\mu$ will be decreasing and eventually zero since

$$
|\hat{N}(s)-1| \leq \int_{1}^{\infty} x^{-\sigma} d|N|(x) \rightarrow 0
$$

as $\sigma \rightarrow \infty$.

Knowledge of the positivity of $\mu$ can be used for locating zeros because of the following result: if $f$ is of finite order in $H_{\beta}$ and has at most finitely many zeros here and $\mu(\sigma)=0$ for $\sigma$ sufficiently large, then $\mu(\sigma)=0$ for $\sigma>\beta$. (This was shown to hold for Beurling zeta functions in [4], but actually the proof readily extends to general functions.) Thus, for example, if $\mu(\sigma)>0$ for $\sigma<\frac{1}{2}$, then $f(s)$ has infinitely many zeros in each half-plane $H_{\frac{1}{2}-\delta}$ for every $\delta>0$.

\section{Main results and proofs}

Suppose $N \in S_{1}$ and $N(x)=x-R(x)$ where $R(x)$ has period 1. Extend $R$ to the whole real line 
by periodicity. Thus $R$ is right continuous, locally of bounded variation, and $R(1)=0$. Since $R$ is of bounded variation, it possesses a Fourier series

$$
a_{0}+\sum_{n=1}^{\infty} b_{n} \cos 2 \pi n x+\sum_{n=1}^{\infty} c_{n} \sin 2 \pi n x
$$

which converges to $\frac{1}{2}(R(x+0)+R(x-0))$, and the series is boundedly convergent (see [5], p.408). Also $b_{n}, c_{n}=O\left(\frac{1}{n}\right)$.

\section{Theorem 1}

Suppose that $N(x)=x-R(x) \in S_{1}$ where $R$ is periodic with period 1. Then $\hat{N}(s)$ has an analytic continuation to $\mathbb{C} \backslash\{1\}$ with a simple pole at $s=1$ with residue 1 . Furthermore $\hat{N}(s)$ is of finite order and for $\sigma<0$ satisfies the relation

$$
\hat{N}(s)=\frac{s}{s-1}+\int_{0}^{1} x^{-s} d R(x)+(2 \pi)^{s} \Gamma(1-s)\left(\cos \frac{\pi s}{2} \sum_{n=1}^{\infty} b_{n} n^{s}-\sin \frac{\pi s}{2} \sum_{n=1}^{\infty} c_{n} n^{s}\right) .
$$

The proof of Theorem 1 shows that the Lindelöf function of $\hat{N}$ satisfies $\mu(\sigma) \leq \frac{1}{2}-\sigma$ for $\sigma \leq 0$, while of course $\mu(\sigma)=0$ for $\sigma \geq 1$. By convexity one obtains upper bounds for all $\sigma$. We can get equality if we know that $b_{n}$ and $c_{n}$ are not identically zero. (Equivalently, since $R$ is right-continuous, if $R$ is not constant; i.e. non-zero.)

\section{Corollary 2}

Under the assumptions of Theorem 1 , if $R \not \equiv 0$ then $\mu(\sigma)=\frac{1}{2}-\sigma$ for $\sigma \leq 0$ and $\mu(\sigma) \geq \mu_{0}(\sigma)$ for all $\sigma$, where

$$
\mu_{0}(\sigma)=\left\{\begin{array}{cc}
0 & \text { if } \sigma \geq \frac{1}{2} \\
\frac{1}{2}-\sigma & \text { if } \sigma<\frac{1}{2}
\end{array} .\right.
$$

It follows that $\hat{N}$ has infinitely many zeros in $H_{\frac{1}{2}-\delta}$ for any $\delta>0$.

In particular, if we let $\Theta$ denote the supremum of the real parts of the zeros of $\hat{N}$, then $\Theta \geq \frac{1}{2}$.

Proof of Theorem 1. We have for $\sigma>1$,

$$
\hat{N}(s)=1+s \int_{1}^{\infty} \frac{N(x)}{x^{s+1}} d x=\frac{s}{s-1}-s \int_{1}^{\infty} \frac{R(x)}{x^{s+1}} d x .
$$

The integral on the right converges for $\sigma>0$, and so $\hat{N}(s)$ has an analytic continuation to $H_{0}$ except for a simple pole at $s=1$ with residue 1 . We can extend further to the left by noting that $a_{0}=\int_{0}^{1} R(x) d x$ so that $\int_{0}^{X}\left(R(x)-a_{0}\right) d x=O(1)$. Hence for $\sigma>0$,

$$
\hat{N}(s)=\frac{s}{s-1}-s \int_{1}^{\infty} \frac{a_{0}}{x^{s+1}} d x-s \int_{1}^{\infty} \frac{R(x)-a_{0}}{x^{s+1}} d x=\frac{s}{s-1}-a_{0}-s \int_{1}^{\infty} \frac{R(x)-a_{0}}{x^{s+1}} d x .
$$

The final integral converges and is holomorphic for $\sigma>-1$ and so this extends $\hat{N}(s)$ holomorphically to $H_{-1}$. Thus $\hat{N}(0)=-a_{0}$. Note that $\hat{N}(s)$ has finite order for $\sigma>-1$ since in this range, writing $V(x)=\int_{1}^{x}\left(R(y)-a_{0}\right) d y=O(1)$, we have

$$
s \int_{1}^{\infty} \frac{R(x)-a_{0}}{x^{s+1}} d x=s(s+1) \int_{1}^{\infty} \frac{V(x)}{x^{s+2}} d x=O\left(|t|^{2}\right) .
$$


Also $s \int_{0}^{1} \frac{R(x)-a_{0}}{x^{s+1}} d x$ converges for $\sigma<0$ and equals $s \int_{0}^{1} \frac{R(x)}{x^{s+1}} d x+a_{0}=\int_{0}^{1} x^{-s} d R(x)+a_{0}$. Thus,

$$
\hat{N}(s)=\frac{s}{s-1}+\int_{0}^{1} x^{-s} d R(x)-s \int_{0}^{\infty} \frac{R(x)-a_{0}}{x^{s+1}} d x \quad \text { for }-1<\sigma<0 .
$$

Now we insert the Fourier series for $R(x)-a_{0}$. If we ignore all problems of convergence for the moment, the final integral of $(2.2)$ becomes

$$
\begin{aligned}
s \int_{0}^{\infty} \frac{R(x)-a_{0}}{x^{s+1}} d x & =s \int_{0}^{\infty} \frac{1}{x^{s+1}}\left(\sum_{n=1}^{\infty} b_{n} \cos 2 \pi n x+\sum_{n=1}^{\infty} c_{n} \sin 2 \pi n x\right) d x \\
& =s \sum_{n=1}^{\infty}\left(b_{n} \int_{0}^{\infty} \frac{\cos 2 \pi n x}{x^{s+1}} d x+c_{n} \int_{0}^{\infty} \frac{\sin 2 \pi n x}{x^{s+1}}\right) d x \\
& =s \sum_{n=1}^{\infty}(2 \pi n)^{s}\left(b_{n} \Gamma(-s) \cos \frac{\pi s}{2}-c_{n} \Gamma(-s) \sin \frac{\pi s}{2}\right) \\
& =-\Gamma(1-s)(2 \pi)^{s}\left(\cos \frac{\pi s}{2} \sum_{n=1}^{\infty} b_{n} n^{s}-\sin \frac{\pi s}{2} \sum_{n=1}^{\infty} c_{n} n^{s}\right)
\end{aligned}
$$

and the result follows formally. However, the term-by-term integration is permissible since the Fourier series is boundedly convergent and $b_{n}$ and $c_{n}$ are both $O(1 / n)$ (the argument is identical to the special case $c_{n}=\frac{1}{n}$ as in [6], p.15).

Thus (2.3) holds for $-1<\sigma<0$. But the RHS of (2.3) is holomorphic for $\sigma<0$. Hence this provides the analytic continuation of $\hat{N}(s)$ to $\mathbb{C} \backslash\{1\}$ and (2.3) holds for $\sigma \leq-1$ also.

That $\hat{N}(s)$ is of finite order follows directly from $(2.3)$. For $\left|\Gamma(1-s)(2 \pi)^{s} \cos \frac{\pi s}{2}\right|=O\left(|t|^{1 / 2-\sigma}\right)$ and similarly for the term involving sin, while $\left|\sum b_{n} n^{s}\right| \leq \sum\left|b_{n}\right| n^{\sigma}=O(1)$ for $\sigma<0$ and also for $\sum c_{n} n^{s}$. Since $\left|\int_{0}^{1} x^{-s} d R(x)\right| \leq \int_{0}^{1} 1 d|R|(x)=O(1)$, (2.3) gives, for $\sigma<0$,

$$
|\hat{N}(\sigma+i t)|=O(1)+O\left(|t|^{1 / 2-\sigma}\right) .
$$

Proof of Corollary 2. Consider the final term in (2.1) which can be written

$$
\Gamma(1-s)(2 \pi)^{s} \cos \frac{\pi s}{2} \sum_{n=1}^{\infty} n^{s}\left(b_{n}-c_{n} \tan \frac{\pi s}{2}\right)
$$

and use the asymptotic bounds

$$
\begin{aligned}
|\Gamma(1-s)| & =|\Gamma(1-\sigma-i t)| \sim \sqrt{2 \pi}|t|^{1 / 2-\sigma} e^{-\frac{\pi}{2}|t|}, \\
\left|\cos \frac{\pi s}{2}\right| & \sim \frac{1}{2} e^{\frac{\pi}{2}|t|}, \quad \text { and } \quad \tan \frac{\pi s}{2}=\tan \left(\frac{\pi \sigma}{2}+i \frac{\pi t}{2}\right)=\operatorname{sgn}(t) i+O\left(e^{-\pi|t|}\right) .
\end{aligned}
$$

Thus the term in (2.4) is, in modulus, asymptotic to

$$
\sqrt{\frac{\pi}{2}}|t|^{1 / 2-\sigma}\left(\left|\sum_{n=1}^{\infty}\left(b_{n} \pm i c_{n}\right) n^{s}\right|+O\left(e^{-\pi|t|}\right)\right) .
$$


Since the coefficients $b_{n}$ and $c_{n}$ are not identically zero and, furthermore, are real, there is a least integer $n_{0}$ for which $b_{n_{0}} \pm i c_{n_{0}} \neq 0$. It follows that for $\sigma$ sufficiently large and negative,

$$
\left|\sum_{n=1}^{\infty}\left(b_{n} \pm i c_{n}\right) n^{s}\right| \geq \frac{1}{2} n_{0}^{\sigma}\left|b_{n_{0}}+i c_{n_{0}}\right| .
$$

This implies that $\mu(\sigma)=\frac{1}{2}-\sigma$ for $\sigma$ sufficiently large and negative. By convexity, $\mu(\sigma) \geq \mu_{0}(\sigma)$ for all $\sigma$. But for $\sigma \leq 0$, we already know that $\mu(\sigma) \leq \frac{1}{2}-\sigma$, so we have equality here.

\section{Remarks}

(a) Theorem 1 and Corollary 2 extend immediately to the case where $N(x)-c x$ is periodic for some constant $c$.

(b) Similar results can be obtained more generally if $R(x)=N(x)-x$ is almost-periodic under some extra assumptions. For example, suppose that

$$
R(x)=a_{0}+\sum_{n=1}^{\infty} b_{n} \cos 2 \pi \lambda_{n} x+\sum_{n=1}^{\infty} c_{n} \sin 2 \pi \lambda_{n} x,
$$

and that the series is boundedly convergent with $b_{n}$ and $c_{n}$ both $O(1 / n)$. Here suppose $\lambda_{n}>0$ increases strictly and without bound. If we assume that $\sum \frac{\lambda_{n}^{\sigma}}{n}$ converges for every $\sigma<0$, then the same method as in Theorem 1 shows that $\hat{N}$ has an analytic continuation to $\mathbb{C} \backslash\{1\}$, is of finite order and satisfies

$$
\hat{N}(s)=\frac{s}{s-1}+\int_{0}^{1} x^{-s} d R(x)+(2 \pi)^{s} \Gamma(1-s)\left(\cos \frac{\pi s}{2} \sum_{n=1}^{\infty} b_{n} \lambda_{n}^{s}-\sin \frac{\pi s}{2} \sum_{n=1}^{\infty} c_{n} \lambda_{n}^{s}\right),
$$

for $\sigma<0$. Corollary 2 also holds in this case if the $b_{n}$ and $c_{n}$ are not identically zero (i.e. $R(x)$ not constant).

(c) The inequality $\mu \geq \mu_{0}$ seems quite robust. It holds for the Beurling zeta function associated to discrete g-prime systems (see [3]) but also for those Mellin transforms contained in (a) and (b) above. What is a natural setting for which this inequality is true?

\section{A particular flow of Mellin transforms to $\zeta(s)$}

As Corollary 2 shows, it is impossible to construct a flow of Mellin transforms with 'periodic' integrator converging to $\zeta(s)$ such that the supremum of the real parts of the zeros converges to $\frac{1}{2}$ from below. Nevertheless, it might still be of interest to investigate a particular flow of such systems with $N(x)-x$ periodic.

Here we consider a particular flow of Mellin transforms $\left\{\hat{N}_{\lambda}(s)\right\}_{\lambda \geq 1}$ converging uniformly to $\zeta(s)$ as $\lambda \rightarrow 1$, and for which $N_{\lambda}(x)-x$ has period 1 with Fourier coefficients proportional to $\frac{1}{n^{\lambda}}$. We shall see that for $\lambda>1, \hat{N}_{\lambda}(s)$ shares a number of characteristics of $\hat{N}_{1}(s)=\zeta(s)$. Thus $\hat{N}_{\lambda}(s)$ has roughly $\frac{T}{2 \pi} \log \frac{T}{2 \pi}-\frac{T}{2 \pi}$ zeros in $H_{0}$ up to height $T$ and an infinite number of negative zeros, roughly at the points $\lambda-1-2 n(n \in \mathbb{N})$.

The Hurwitz zeta function $\zeta(s, a)$, defined for $\Re s>1$ and $0<a \leq 1$ by the series $\sum_{n=0}^{\infty}(n+$ $a)^{-s}$ has (as a function of $s$ ) an analytic continuation to $\mathbb{C} \backslash\{1\}$ and a simple pole at $s=1$ 
with residue 1 (see for example [1], Chapter 12). Its analytic continuation is given by $\zeta(s, a)=$ $\Gamma(1-s) I(s, a)$, where $I(s, a)$ is the entire function

$$
I(s, a)=\frac{1}{2 \pi i} \int_{C} \frac{z^{s-1} e^{a z}}{1-e^{z}} d z
$$

where $C$ is the contour which starts at $-\infty$, goes along the negative real axis (on the lower side) to $-c$ where $0<c<2 \pi$, encircles the origin back to $-c$ and returns to $-\infty$ on the upper side of the negative real axis. Note that $\zeta(s, 1)=\zeta(s)$. The definition actually makes sense whenever $\Re a>0$ (any $s$ ). As a function of $a$ (for any given $s$ ), $I(s, a)$ is holomorphic for $\Re a>0$.

Definition: Let $N_{\lambda}(x)=x-R_{\lambda}(x)$ for $x \geq 1$ and zero otherwise and $\lambda \geq 1$, where $R_{\lambda}(x)$ is periodic with period 1 and be defined for $0 \leq x<1$ by

$$
R_{\lambda}(x)=\rho_{\lambda}(\zeta(1-\lambda, 1-x)-\zeta(1-\lambda))=\frac{\rho_{\lambda} \Gamma(\lambda)}{2 \pi i} \int_{C} \frac{z^{-\lambda}\left(e^{-x z}-1\right)}{e^{-z}-1} d z .
$$

Here $\rho_{\lambda}$ is a continuous function of $\lambda$ (to be determined) and we set $\rho_{1}=1$ so that $R_{1}(x)=\{x\}$.

\section{Some properties}

(a) For $\lambda=m \in \mathbb{N}, R_{m}$ is a polynomial in $[0,1)$ since $\zeta(-n, a)=-\frac{B_{n+1}(a)}{n+1}$ where $B_{n}(\cdot)$ is the $n^{\text {th }}$ Bernoulli polynomial; i.e.

$$
R_{m}(x)=\frac{\rho_{m}}{m}\left(B_{m}(1)-B_{m}(1-x)\right)=\frac{(-1)^{m-1} \rho_{m}}{m}\left(B_{m}(x)-B_{m}(0)\right) . \quad(0 \leq x<1)
$$

(b) For $\lambda>1 R_{\lambda}$ is continuous, while $R_{1}$ is right continuous but has jump continuities at the integers. On the interval $[0,1), R_{\lambda}$ is holomorphic since the function

$$
R_{\lambda}^{*}(z)=\rho_{\lambda}(\zeta(1-\lambda, 1-z)-\zeta(1-\lambda))
$$

which agrees with $R_{\lambda}$ on $[0,1)$, is holomorphic for $\Re z<1$. Hence we have an expansion

$$
R_{\lambda}(x)=\sum_{n=1}^{\infty} a_{n}(\lambda) x^{n} \quad(0 \leq x<1)
$$

for some coefficients $a_{n}(\lambda)$. Expanding the integrand in (3.1) gives a formula for the coefficients.

$$
\begin{aligned}
R_{\lambda}(x) & =\frac{\rho_{\lambda} \Gamma(\lambda)}{2 \pi i} \int_{C} \frac{z^{-\lambda}}{e^{-z}-1} \sum_{n=1}^{\infty}(-1)^{n} \frac{x^{n} z^{n}}{n !} d z=\sum_{n=1}^{\infty} \frac{(-1)^{n}}{n !}\left(\frac{\rho_{\lambda} \Gamma(\lambda)}{2 \pi i} \int_{C} \frac{z^{n-\lambda}}{e^{-z}-1} d z\right) x^{n} \\
& =\sum_{n=1}^{\infty} \frac{(-1)^{n}}{n !} \frac{\rho_{\lambda} \Gamma(\lambda) \zeta(n-\lambda+1)}{\Gamma(\lambda-n)} x^{n} .
\end{aligned}
$$

Hence

$$
a_{n}(\lambda)=(-1)^{n} \rho_{\lambda}\left(\begin{array}{c}
\lambda-1 \\
n
\end{array}\right) \zeta(n+1-\lambda)
$$

For $\lambda>1$ the expansion is also valid for $x=1$, since $a_{n}(\lambda)=O\left(n^{-\lambda}\right)$. For $\lambda=m \in \mathbb{N}$ and $n=m,(3.2)$ should be interpreted as $\lim _{\lambda \rightarrow m} a_{m}(\lambda)=(-1)^{m-1} \rho_{m} / m$. Of course in this case the expansion is finite and is a polynomial of degree $m$. 
(c) Fourier expansion: We have

$$
R_{\lambda}(x)=-\frac{2 \rho_{\lambda} \Gamma(\lambda)}{(2 \pi)^{\lambda}}\left(\cos \frac{\pi \lambda}{2} \sum_{n=1}^{\infty} \frac{1-\cos 2 \pi n x}{n^{\lambda}}+\sin \frac{\pi \lambda}{2} \sum_{n=1}^{\infty} \frac{\sin 2 \pi n x}{n^{\lambda}}\right)
$$

which holds for all $x \in \mathbb{R}$ if $\lambda>1$ and for $x \in \mathbb{R} \backslash \mathbb{Z}$ if $\lambda=1$ ([1], p.257).

By Theorem $1, \hat{N}_{\lambda}$ extends analytically to the complex plane except for a simple pole at 1 and (after some calculation)

$$
\hat{N}_{\lambda}(s)=\frac{s}{s-1}+\int_{0}^{1} x^{-s} d R_{\lambda}(x)+2 \rho_{\lambda}(2 \pi)^{s-\lambda} \Gamma(\lambda) \Gamma(1-s) \cos \frac{\pi(s-\lambda)}{2} \zeta(\lambda-s) .
$$

Using the functional equation for $\zeta(\lambda-s)$ this becomes

$$
\hat{N}_{\lambda}(s)=\frac{s}{s-1}+\int_{0}^{1} x^{-s} d R_{\lambda}(x)+\rho_{\lambda} \frac{\Gamma(\lambda) \Gamma(1-s)}{\Gamma(\lambda-s)} \zeta(s-\lambda+1) .
$$

For $\lambda>1$ we have for $\sigma<1$,

$$
\int_{0}^{1} x^{-s} d R_{\lambda}(x)=\int_{0}^{1} x^{-s} R_{\lambda}^{\prime}(x) d x=\int_{0}^{1} \sum_{n=1}^{\infty} n a_{n}(\lambda) x^{n-s-1} d x=\sum_{n=1}^{\infty} \frac{n a_{n}(\lambda)}{n-s} .
$$

This series converges for all $s \notin \mathbb{N}$ and provides the meromorphic continuation of the LHS to $\mathbb{C}$ with (at most) simple poles at the positive integers. Thus (3.3)-(3.5) hold for all $s$.

\section{Theorem 3}

With $N_{\lambda}$ as defined above, we have $\hat{N}_{\lambda}(s) \rightarrow \zeta(s)$ as $\lambda \rightarrow 1$ uniformly on compact subsets of $\mathbb{C} \backslash\{1\}$.

Proof. This basically follows from the fact that $R_{\lambda} \rightarrow R_{1}$ uniformly on $[0, a]$ for every $a<1$, but we need to be a little careful near 1 since $R_{1}$ is not continuous here. First consider $\sigma>0$. Let $K$ be a compact subset of $H_{0} \backslash\{1\}$. We have for $s \in K$

$$
\left|\hat{N}_{\lambda}(s)-\hat{N}_{1}(s)\right|=\left|s \int_{1}^{\infty} \frac{R_{\lambda}(x)-R_{1}(x)}{x^{s+1}} d x\right| \leq A \int_{1}^{\infty} \frac{\left|R_{\lambda}(x)-R_{1}(x)\right|}{x^{\sigma_{0}+1}} d x
$$

for some constants $A, \sigma_{0}>0$. Let $\eta>0$. Then for all $\varepsilon>0$, there exists $\lambda_{0}$ such that for $1<\lambda<\lambda_{0},\left|R_{1}(x)-R_{\lambda}(x)\right|<\varepsilon$ for $n \leq x \leq n+1-\eta$ (any $n \in \mathbb{Z}$ ). Hence

$$
\left|\hat{N}_{\lambda}(s)-\hat{N}_{1}(s)\right| \leq A \varepsilon \int_{1}^{\infty} \frac{1}{x^{\sigma_{0}+1}} d x+A \sum_{n=1}^{\infty} \int_{n+1-\eta}^{n+1} \frac{C}{x^{\sigma_{0}+1}} d x \leq A_{1} \varepsilon+A C \eta \sum_{n=1}^{\infty} \frac{1}{n^{\sigma_{0}+1}}
$$

which can be made as small as we please. Hence $\hat{N}_{\lambda}(s) \rightarrow \hat{N}_{1}(s)$ uniformly on compact subsets of $H_{0} \backslash\{1\}$.

In fact the same argument works for compact subsets of $H_{-1} \backslash\{1\}$ if we use the expression

$$
\hat{N}_{\lambda}(s)=\frac{s}{s-1}-a_{0}+s(s+1) \int_{1}^{\infty} \frac{V_{\lambda}(x)}{x^{s+2}} d x
$$

where $V_{\lambda}(x)=\int_{1}^{x}\left(R_{\lambda}(\cdot)-a_{0}\right)$, and noting that $V_{\lambda} \rightarrow V_{1}$ uniformly. 
For $\sigma<0$ we can use (3.4). The final term tends locally uniformly to $\zeta(s)$, while

$$
\int_{0}^{1} x^{-s} d R_{\lambda}(x)=s \int_{0}^{1} \frac{R_{\lambda}(x)}{x^{s+1}} d x \rightarrow s \int_{0}^{1} \frac{R_{1}(x)}{x^{s+1}} d x=-\frac{s}{s-1},
$$

the convergence again being uniform. The result now follows.

\section{Zeros}

Since $\hat{N}_{\lambda}(s) \rightarrow \zeta(s)$ locally uniformly, the Riemann Hypothesis will follow if we can show that for all $\lambda$ close to 1 (with some particular choice of $\rho_{\lambda}$ ), $\hat{N}_{\lambda}(s)$ has no zeros with $\sigma>\frac{1}{2}$. Slightly less restrictively, $\mathrm{RH}$ is true if the following conjecture is true:

Conjecture: Given $\theta>\frac{1}{2}$, there exists $\lambda_{\theta}>1$ such that for $1<\lambda<\lambda_{\theta}$ and some suitable choice of $\rho_{\lambda}, \hat{N}_{\lambda}$ has no zeros in $H_{\theta}$.

It may even be the case that this conjecture is equivalent to $\mathrm{RH}$. The hope is of course that it is easier to show that for $\lambda>1, \hat{N}_{\lambda}$ has no zeros in $H_{\theta}$ than it is for $\lambda=1$.

Now we show that for $\lambda>\frac{3}{2}, \hat{N}_{\lambda}$ has only finitely many zeros in $H_{\frac{1}{2}+\delta}$ (any $\delta>0$ ). As $\lambda$ gets closer to 1 however, we can only be certain of having finitely many zeros in half-planes further to the right, since we do not have the strong bounds on $\zeta$ in vertical strips. If we assume the Lindelöf Hypothesis $(\mathrm{LH})$, then $\hat{N}_{\lambda}$ has only finitely many zeros in $H_{\frac{1}{2}+\delta}$ for every $\lambda>1$.

\section{Theorem 4}

(i) Let $\lambda \geq \frac{3}{2}$. Then for every $\delta>0, \hat{N}_{\lambda}(s)$ has at most finitely many zeros in $H_{\frac{1}{2}+\delta}$ and in every strip where $\sigma \in\left[-A, \frac{1}{2}-\delta\right]$ (any $A$ ).

(ii) Let $1<\lambda<\frac{3}{2}$. Then for every $\delta>0, \hat{N}_{\lambda}(s)$ has at most finitely many zeros in $H_{2-\lambda+\delta}$ $\left(H_{\frac{1}{2}+\delta}\right.$ on $\left.L H\right)$ and in every strip where $\sigma \in[-A, \lambda-1-\delta]\left(\sigma \in\left[-A, \frac{1}{2}-\delta\right]\right.$ on $\left.R H\right)$.

Proof. For $\lambda>1, \int_{0}^{1} x^{-s} d R_{\lambda}(x)=\sum_{n=1}^{\infty} \frac{n a_{n}(\lambda)}{n-s} \rightarrow 0$ as $|t| \rightarrow \infty$ for every $\sigma$. Hence from (3.4),

$$
\hat{N}_{\lambda}(\sigma+i t)=1+o(1)+\rho_{\lambda} \frac{\Gamma(\lambda) \Gamma(1-\sigma-i t)}{\Gamma(\lambda-\sigma-i t)} \zeta(\sigma-\lambda+1+i t) .
$$

The term on the right is, in modulus, asymptotic to

$$
\left|\rho_{\lambda}\right| \Gamma(\lambda) \frac{|\zeta(\sigma-\lambda+1+i t)|}{|t|^{\lambda-1}}=O\left(|t|^{\mu(\sigma-\lambda+1)-\lambda+1+\varepsilon}\right),
$$

for every $\varepsilon>0$, where $\mu(\cdot)$ is the Lindelöf function for $\zeta$. Note that the implied constant is independent of $\sigma$ for $a \leq \sigma \leq b$, any $a, b$.

Let $\lambda>\frac{3}{2}$. Consider $\sigma \leq \lambda-1$ and $\sigma>\lambda-1$ separately. If $\sigma \leq \lambda-1$, then $\mu(\sigma-\lambda+1)=$ $\lambda-\sigma-\frac{1}{2}$, and the exponent of $|t|$ in (3.6) is $\frac{1}{2}-\sigma+\varepsilon$. This is negative (for sufficiently small $\varepsilon$ ) if $\sigma>\frac{1}{2}$. If $\sigma>\lambda-1, \mu(\sigma-\lambda+1)<\frac{1}{2}$, so the exponent is also negative for $\varepsilon$ small enough. Since the bound is uniform in $\sigma$, and there are no zeros in $H_{A}$ for $A$ sufficiently large, this implies that for $\lambda \geq \frac{3}{2}, \hat{N}_{\lambda}$ has only finitely many zeros in $H_{\frac{1}{2}+\delta}$ for each $\delta>0$.

If $\sigma<\frac{1}{2}$, then $\sigma<\lambda-1$ and the expression in (3.6) is at least ${ }^{2}$

$$
c|t|^{\frac{1}{2}-\sigma}
$$

\footnotetext{
${ }^{2}$ Assuming $\rho_{\lambda} \neq 0$. If $\rho_{\lambda}=0$, the result is trivially true.
} 
for some $c>0$, depending continuously on $\lambda$ and $\sigma$. Hence for $-A \leq \sigma \leq \frac{1}{2}-\delta$, this is at least $c_{1}|t|^{\delta}$ (some constant $c_{1}>0$ ) which tends to infinity. Thus there are no zeros with $|t|$ sufficiently large in such a strip, proving assertion (i).

Now consider $1<\lambda<\frac{3}{2}$. If $\sigma \geq \lambda$, then $\mu(\sigma-\lambda+1)=0$ and the exponent in (3.6) is negative. For $\lambda-1 \leq \sigma<\lambda, \mu(\sigma-\lambda+1) \leq \frac{\lambda-\sigma}{2}$ (using $\mu(\alpha) \leq \frac{1-\alpha}{2}$ for $0 \leq \alpha \leq 1$ ) and the exponent in (3.6) is $1-\frac{\lambda+\sigma}{2}+\varepsilon$. This is negative for $\sigma>2-\lambda$, and the result follows.

If L.H. holds, then $\mu(\sigma-\lambda+1)=0$ for $\sigma>\lambda-\frac{1}{2}$ and $\mu(\sigma-\lambda+1)=\lambda-\sigma-\frac{1}{2}$ for $\sigma \leq \lambda-\frac{1}{2}$. Hence the exponent in (3.6) is now

$$
\begin{array}{ll}
1-\lambda+\varepsilon & \text { if } \sigma>\lambda-\frac{1}{2} \\
\frac{1}{2}-\sigma+\varepsilon & \text { if } \sigma \leq \lambda-\frac{1}{2}
\end{array}
$$

Both are negative if $\sigma>\frac{1}{2}$ for sufficiently small $\varepsilon$.

As in part(i), if $\sigma<\lambda-1$, then $\sigma-\lambda+1<0$ and the expression in (3.6) is at least $c|t|^{\frac{1}{2}-\sigma} \rightarrow \infty$. For $\sigma \geq \lambda-1$ we cannot deduce anything about (3.6) for large $|t|$ unless we know that $\zeta$ has no zeros in certain strips inside the critical strip. On R.H., the above argument applies for $\sigma-\lambda+1<\frac{1}{2}$, and (ii) follows.

Remark. For $\lambda>\frac{3}{2}$, the zeros in any right half-plane (apart from at most a finite number of exceptions) actually lie in a region

$$
\left\{\sigma+i t:-\frac{A}{\log |t|} \leq \sigma-\frac{1}{2} \leq \frac{B}{\log |t|},|t| \geq 2\right\}
$$

for some constants $A, B$. For $\hat{N}_{\lambda}(s)=0$ if and only if

$$
\frac{s}{s-1}+\int_{0}^{1} x^{-s} d R_{\lambda}(x)=-2 \rho_{\lambda}(2 \pi)^{s-\lambda} \Gamma(\lambda) \Gamma(1-s) \cos \frac{\pi(s-\lambda)}{2} \zeta(\lambda-s) .
$$

Take $\sigma$ such that $\left|\sigma-\frac{1}{2}\right| \leq \lambda-\frac{3}{2}-\delta$ for some $\delta>0$, and $|t| \geq 2$. The LHS of (3.7) is $1+o(1)$, while the RHS is, in modulus,

$$
\sim \frac{\left|\rho_{\lambda}\right| \Gamma(\lambda)}{(2 \pi)^{\lambda-\sigma-\frac{1}{2}}}|t|^{\frac{1}{2}-\sigma}|\zeta(\lambda-\sigma-i t)| .
$$

Since $\lambda-\sigma \geq 1+\delta$, this is $\asymp|t|^{\frac{1}{2}-\sigma}$, uniformly in $\sigma$. In particular, for $\frac{1}{2}-\sigma>A / \log |t|$ and $A$ sufficiently large, the LHS of (3.7) is less than the RHS in modulus, and hence there are no zeros for $|t|$ sufficiently large in this range. Similarly, for $\sigma-\frac{1}{2}>B / \log |t|$ and $B$ sufficiently large, the LHS is greater than the RHS in modulus.

We can be more precise. Let $\sigma=\frac{1}{2}+\frac{\theta_{t}}{\log |t|}$ where $\theta_{t}=O(1)$. Then for a zero $\sigma+i t$ with large $|t|$, we need

$$
\frac{\left|\rho_{\lambda}\right| \Gamma(\lambda)}{(2 \pi)^{\lambda-1}} e^{-\theta_{t}}|\zeta(\lambda-\sigma-i t)| \sim 1 .
$$

Since $|\zeta(\lambda-\sigma-i t)| \sim\left|\zeta\left(\lambda-\frac{1}{2}-i t\right)\right|$, this requires

$$
\theta_{t}=\log \left(\frac{\left|\rho_{\lambda}\right| \Gamma(\lambda)}{(2 \pi)^{\lambda-1}}\left|\zeta\left(\lambda-\frac{1}{2}-i t\right)\right|\right)+o(1)
$$


As such and taking $t \geq 2$, the RHS of (3.7) is, using Stirling's formula, asymptotically

$$
-\frac{\rho_{\lambda} \Gamma(\lambda)}{(2 \pi)^{\lambda-1}} e^{\theta_{t}+\frac{i \pi}{2}\left(\lambda-\frac{1}{2}\right)} e^{-i(t \log t-t-t \log 2 \pi)} \zeta\left(\lambda-\frac{1}{2}-i t\right)=-\frac{\rho_{\lambda} \zeta\left(\lambda-\frac{1}{2}-i t\right)}{\left|\rho_{\lambda} \zeta\left(\lambda-\frac{1}{2}-i t\right)\right|} e^{-i\left(t \log t-t-t \log 2 \pi-\frac{\pi}{2}\left(\lambda-\frac{1}{2}\right)\right)} .
$$

At a zero, we want this to be asymptotic to the LHS of (3.7); i.e. to 1 . Thus we want $t \log t-$ $t-t \log 2 \pi=2 \pi k+O(1)$ for $k \in \mathbb{Z}$; i.e.

$$
f(t):=\frac{t}{2 \pi} \log \frac{t}{2 \pi}-\frac{t}{2 \pi}=k+O(1)
$$

Since $f(t)$ is continuous we should expect a zero $\sigma_{k}+i t_{k}$ for each $k$ sufficiently large. The number of such zeros with $t_{k} \leq T$ is therefore roughly $f(T)$; i.e. we should expect, for $\lambda>\frac{3}{2}$,

$$
\frac{T}{2 \pi} \log \frac{T}{2 \pi}-\frac{T}{2 \pi}+O(1)
$$

zeros up to height $T$.

\section{Theorem 5}

Let $\lambda>1$. Then $\hat{N}_{\lambda}$ has

$$
\frac{T}{2 \pi} \log \frac{T}{2 \pi}-\frac{T}{2 \pi}+O(\log T)
$$

zeros in the rectangular strip $\{\sigma+i t: 0 \leq \sigma \leq 1,0 \leq t \leq T\}$.

Proof. Choose $\sigma_{0}$ sufficiently large so that $\left|\Re \hat{N}_{\lambda}\left(\sigma_{0}+i t\right)\right| \geq c>0$ for all $t$.

Denote by $n(T)$ the number of zeros in the rectangular strip

$$
\left\{\sigma+i t: 0 \leq \sigma \leq \sigma_{0}, 1 \leq t \leq T\right\} .
$$

This differs from the required number by $O(1)$. Let $\gamma$ denote the (anti-clockwise) boundary path of this strip. We may assume without loss of generality that there are no zeros of $\hat{N}_{\lambda}$ on $\gamma$. Then

$$
n(T)=\frac{1}{2 \pi} \Delta_{\gamma} \arg \hat{N}_{\lambda},
$$

where $\Delta_{\gamma} \arg \hat{N}_{\lambda}$ is the continuous variation of the argument of $\hat{N}_{\lambda}$ around $\gamma$.

On the right-hand vertical, $\hat{N}_{\lambda}\left(\sigma_{0}+i t\right) \rightarrow 1$ as $t \rightarrow \infty$. Hence the variation of the argument along this vertical line segment is $O(1)$. For the top horizontal, we use Lemma 9.4 of [6] (with ' 2 ' replaced by ' $\sigma_{0}$ '). Since $\hat{N}_{\lambda}$ has finite order, this Lemma implies that the variation along here is at most $O(\log T)$. The variation along the bottom horizontal is trivially $O(1)$. Finally on the left vertical, we have

$$
\begin{aligned}
\hat{N}_{\lambda}(i t) & =2 \rho_{\lambda} \Gamma(\lambda)(2 \pi)^{i t-\lambda} \Gamma(1-i t) \cos \frac{\pi(i t-\lambda)}{2} \zeta(\lambda-i t)+1+o(1) \\
& \sim \frac{\rho_{\lambda} \Gamma(\lambda)}{(2 \pi)^{\lambda-\frac{1}{2}}} t^{\frac{1}{2}} e^{-i(t \log t-t-t \log 2 \pi)} e^{\frac{i \pi}{2}\left(\lambda-\frac{1}{2}\right)} \zeta(\lambda-i t) .
\end{aligned}
$$

Since $\zeta(\lambda-i t)$ is bounded and bounded away from zero, $\arg \hat{N}_{\lambda}(i t)=-(t \log t-t-t \log 2 \pi)+O(1)$, and the variation of the argument along the (downward) left hand vertical is $T \log T-T-$ $T \log 2 \pi+O(1)$. 
Remark. It seems plausible that the $O(\log T)$-term can be replaced by $O\left((\log T)^{\kappa}\right)$, with $\kappa$ decreasing steadily from 1 to 0 as $\lambda$ varies from 1 to $\frac{3}{2}$.

Zeros on the negative real axis: For $\lambda=1, \hat{N}_{\lambda}(s)=\zeta(s)$ has zeros on the negative real axis at $-2 k$ for each positive integer $k$ - the so-called trivial zeros. Very similar behaviour occurs for $\lambda>1$.

We require the following elementary result.

\section{Lemma 6}

Suppose $f$ is holomorphic and real valued on $[0, \infty)$. Suppose further that, as $x \rightarrow \infty$,

$$
f(x)=\cos \frac{\pi x}{2}+o(1) \quad \text { and } \quad f^{\prime}(x)=-\frac{\pi}{2} \sin \frac{\pi x}{2}+o(1) .
$$

Then for every sufficiently large integer $n$, the interval $(2 n, 2 n+2)$ contains exactly one zero, say $x_{n}$, and $x_{n}=2 n+1+o(1)$.

Proof. For $n \in \mathbb{N}, f(2 n)-(-1)^{n} \rightarrow 0$, so for $n$ sufficiently large, the sign of $f(2 n)$ is $(-1)^{n}$. Hence there is at least one zero in each interval $(2 n, 2 n+2)$ (for $n$ large). In fact the zero(s) must be close to $2 n+1$ since for $|h| \leq 1$,

$$
f(2 n+h)-(-1)^{n} \cos \frac{\pi h}{2} \rightarrow 0
$$

uniformly in $h$, and $\cos \frac{\pi h}{2}$ is bounded away from zero if $|h|<1$.

Now for $x=2 n+y, f^{\prime}(x)=(-1)^{n-1} \frac{\pi}{2} \sin \frac{\pi y}{2}+o(1)$, so for $x \in[2 n+h, 2 n+2-h]$ (any fixed $h>0),(-1)^{n-1} f^{\prime}(x)>0$ for $n$ large enough; i.e. $f$ is monotonic in this interval. Thus can be at most one zero, say $x_{n}$. This must satisfy $x_{n}=2 n+1+o(1)$.

\section{Theorem 7}

For every sufficiently large positive integer $n, \hat{N}_{\lambda}(\lambda-x)$ has exactly one zero $x_{n}$ in each interval $(2 n, 2 n+2)(n \in \mathbb{N})$. Furthermore $x_{n}=2 n+1+o(1)$ as $n \rightarrow \infty$.

Proof. Apply Lemma 6 with

$$
f(x)=\frac{(2 \pi)^{x} \hat{N}_{\lambda}(\lambda-x)}{2 \rho_{\lambda} \Gamma(\lambda) \Gamma(x+1-\lambda)}=\zeta(x) \cos \frac{\pi x}{2}+\frac{(2 \pi)^{x}}{2 \rho_{\lambda} \Gamma(\lambda) \Gamma(x+1-\lambda)}\left(\frac{x}{x+1}+\sum_{m=1}^{\infty} \frac{m a_{\lambda}(m)}{m+x}\right)
$$

(using (3.5)). The final term and its derivarive tend to 0 with $x$, while $\zeta(x) \rightarrow 1, \zeta^{\prime}(x) \rightarrow 0$, so $f$ satisfies the conditions of Lemma 6 and the result follows.

Acknowledgement. It is a pleasure for me to thank Eric Saias for some stimulating discussions on flows of Mellin transforms to the Riemann zeta function. 


\section{References}

[1] T. M. Apostol, Introduction to Analytic Number Theory, Springer, 1976.

[2] P. T. Bateman and H. G. Diamond, Analytic Number Theory, World Scientific Publishing, 2004.

[3] T. W. Hilberdink, A lower bound for the Lindelöf function associated to generalised integers, $J$. Number Theory 122 (2007) 336-341.

[4] T. W. Hilberdink and M. L. Lapidus, Beurling zeta functions, Generalised Primes, and Fractal Membranes, Acta Appl Math 94 (2006) 21-48.

[5] E. C. Titchmarsh, The Theory of Functions, Second edition, Oxford University Press, 1986.

[6] E. C. Titchmarsh, The Theory of the Riemann Zeta-function, Second edition, Oxford University Press, 1986. 